\title{
COMPARAÇÃO ENTRE A COMPOSIÇÃO QUÍMICA E CAPACIDADE ANTIOXIDANTE DE DIFERENTES EXTRATOS DE PRÓPOLIS VERDE
}

\author{
Fernanda B. Salgueiro e Rosane N. Castro* \\ Departamento de Química, Instituto de Ciências Exatas, Universidade Federal Rural do Rio de Janeiro, 23897-000 Seropédica - \\ RJ, Brasil
}

Recebido em 03/04/2016; aceito em 27/05/2016; publicado na web em 01/08/2016

\begin{abstract}
COMPARISON BETWEEN CHEMICAL COMPOSITION AND ANTIOXIDANT ACTIVITY OF DIFFERENT EXTRACTS OF GREEN PROPOLIS. In this work were determined the total phenolic contents, antioxidant activity and chemical composition of twelve samples of green propolis acquired from beekeepers and other twelve commercial extracts samples from different regions of Southeast Brazil. The phenolic contents and the antioxidant activity were evaluated by the Folin-Ciocalteau and DPPH, ABTS and FRAP methods, respectively. Both types of propolis showed significant radical scavenging properties. HPLC-PDA was applied for quantification of chlorogenic acid, caffeic acid, ferulic acid, para-coumaric acid, rosmarinic acid, vanillin, hesperidin, naringenin, pinobanksin, kaempferol, Artepillin-C (4-hydroxy-3,5-diprenyl cinnamic acid), kampheride and pinostrobin. Despite the chemical composition of both in natura and commercial propolis extracts were similar the multivariate analysis allowed the discrimination between them.
\end{abstract}

Keywords: green propolis; commercial extract; polyphenols; chemometrics.

\section{INTRODUÇÃO}

Própolis é uma mistura complexa de substâncias resinosas, gomosas e balsâmicas coletadas pelas abelhas melíferas de brotos, flores e exsudados de plantas, às quais as abelhas acrescentam secreções salivares, cera e pólen para a elaboração do produto final. A própolis bruta encontra-se no estado sólido, sendo dura a $15{ }^{\circ} \mathrm{C}$ e maleável a partir $\operatorname{dos} 30^{\circ} \mathrm{C}$. Suas propriedades físicas, como cor, odor e faixa de fusão $\left(60{ }^{\circ} \mathrm{C}-70{ }^{\circ} \mathrm{C}\right)$ variam de uma amostra para outra. Devido à grande diversidade de espécies vegetais brasileiras visitadas pelas abelhas, ocorre uma elevada variação de seus princípios ativos. Sua cor varia do verde, vermelho ao marrom escuro, e esta variação está relacionada com a origem geográfica e a vegetação de onde ela é extraída. ${ }^{1}$

Estudos realizados mostram que mais de trezentas substâncias foram identificadas em amostras de própolis, das quais se destacam: os flavonoides (galangina, crisina, tectocrisina, pinocembrina, canferol e quercetina), além dos aldeídos aromáticos (vanilina e isovanilina), cumarinas, ácidos fenólicos (ácido caféico, ferúlico, cinâmico e cumárico), ácidos orgânicos (ácido benzóico), ácidos e ésteres alifáticos e aromáticos, açúcares, alcoóis, ácidos graxos, aminoácidos, esteroides, cetonas, chalconas e diidrochalconas, terpenoides e proteínas. ${ }^{2}$ Dentre estas classes de substâncias, destacam-se a dos flavonoides e a dos ácidos fenólicos, pois são atribuídas a elas grandes parte das atividades biológicas descritas para a própolis. Os flavonoides e ácidos fenólicos são as principais classes de substâncias fenólicas, cujas relações estrutura-atividade antioxidante em sistemas aquosos ou lipofílicos têm sido extensivamente relatadas. ${ }^{3}$ Ocorrendo naturalmente, espera-se que esses polifenóis ajudem a reduzir o risco de várias doenças, incluindo câncer e doenças cardiovasculares, devido à sua atividade antioxidante.

Há um interesse pelo consumo de própolis crescente no Brasil e no mundo devido ao aumento nas pesquisas envolvendo sua composição química e propriedades terapêuticas. Existem vários estudos

*e-mail: nora@ufrrj.br

"This paper is part of the PubliSBQ Special Issue in honor of the late Prof. Angelo da Cunha Pinto. que atribuem à própolis diversas aplicações na medicina popular, os quais relatam seu grande potencial terapêutico. Diversas propriedades biológicas, tais como antimicrobiana, anti-inflamatória, cicatrizante, anestésica, anticarcinogênica, antiviral, antioxidante e fitotóxica têm sido atribuídas à própolis e aos seus constituintes. ${ }^{4}$

O Brasil está em terceiro lugar no ranking mundial em termos de produção da própolis. As condições climáticas, geográficas e as características da vegetação favorecem esta produção que tem sua maior demanda voltada para o mercado japonês. De toda a própolis in natura consumida no Japão, cerca de $92 \%$ é de origem brasileira e, segundo os dados da Japan Trade Organization, o frasco do extrato alcoólico da substância é vendido por até US\$110.00. ${ }^{5}$

Própolis possui atividade antioxidante, seus constituintes são capazes de sequestrar os radicais livres. Por outro lado, a sua composição química e, portanto, a atividade antioxidante, pode variar amplamente de acordo com a localização, época da colheita ou simplesmente comparando com outra colmeia. Apesar de existir uma série de estudos comparativos avaliando a capacidade antioxidante da própolis, poucos são os relatos sobre amostras do estado do Rio de Janeiro. O propósito desse estudo foi determinar a composição química, quanto aos fenólicos e flavonoides totais, e testar e comparar a capacidade antioxidante de doze amostras de própolis verde bruta e doze amostras de tinturas de própolis comercializadas em diferentes regiões do sudeste do Brasil. Os extratos etanólicos de própolis foram analisados por CLAE-DAD para determinar e quantificar as substâncias bioativas responsáveis por sua propriedade antioxidante e, assim, entender a relação entre a composição e a atividade antioxidante. Além disso, a técnica de análise multivariada (PCA) foi aplicada ao conjunto de dados obtidos com as análises dos extratos etanólicos da própolis in natura e nas tinturas comerciais, para efeitos de comparação e para avaliação da qualidade dos extratos comerciais.

\section{PARTE EXPERIMENTAL}

\section{Amostras de própolis}

As doze amostras de própolis verde bruta (E) de Apis mellifera 
foram obtidas diretamente de diferentes apicultores, enquanto as doze tinturas de própolis (C) foram adquiridas atráves de compra em lojas especializadas no comércio de distintas cidades do estado do Rio de Janeiro, sendo que algumas dessas amostras são produzidas em outros estados da região sudeste (Tabela 1). As amostras in natura foram pulverizadas em liquidificador, mantidas em frascos bem fechados e armazenadas em freezer até o momento das extrações.

Tabela 1. Amostras de própolis bruta e extratos comerciais estudados

\begin{tabular}{|c|c|c|}
\hline Amostras & Origem Geográfica & Data de Coleta \\
\hline E1 & Seropédica - RJ & Julho/2010 \\
\hline E2 & Mesquita - RJ & Julho - 2012 \\
\hline $\mathbf{E 3}$ & Seropédica - RJ & Julho-2012 \\
\hline E4 & Cacaria - RJ & Agosto -2012 \\
\hline E5 & Carmo - RJ & Setembro - 2012 \\
\hline E6 & Cordeiro - RJ & Março - 2012 \\
\hline E7 & Paracambi - RJ & Setembro - 2012 \\
\hline E8 & Cacaria - RJ & Outubro - 2012 \\
\hline E9 & Cacaria - RJ & Outubro - 2012 \\
\hline E10 & Cacaria - RJ & Outubro - 2012 \\
\hline E11 & Cacaria - RJ & Outubro - 2012 \\
\hline E12 & Paracambi - RJ & Novembro - 2012 \\
\hline C1 & Rio de Janeiro & Março - 2012 \\
\hline $\mathrm{C} 2$ & Rio de Janeiro & Abril - 2012 \\
\hline $\mathbf{C 3}$ & Rio de Janeiro & Julho - 2012 \\
\hline $\mathrm{C} 4$ & São Paulo & Maio - 2012 \\
\hline $\mathrm{C5}$ & Rio de Janeiro & Outubro - 2011 \\
\hline C6 & Rio de Janeiro & Fevereiro - 2012 \\
\hline C7 & Rio de Janeiro & Abril - 2012 \\
\hline C8 & Rio de Janeiro & Março - 2012 \\
\hline C9 & São Paulo & Junho - 2012 \\
\hline C10 & Minas Gerais & Junho - 2012 \\
\hline C11 & Rio de Janeiro & Julho - 2012 \\
\hline $\mathrm{C} 12$ & Rio de Janeiro & Julho - 2012 \\
\hline
\end{tabular}

$\mathbf{E}$ - própolis bruta $\mathbf{C}$ - extratos comerciais.

\section{Reagentes}

Os reagentes carbonato de sódio anidro, cloreto de alumínio hexaidratado, bissulfito de sódio, assim como o metanol e ácido acético grau espectroscópico foram comprados da VETEC (RJ, Brasil). Os reagentes DPPH (2,2-difenil-1-picril-hidrazil), ABTS [2,2'-azinobis(3-etilbenzotiazolina-6-ácido sulfônico)], TPTZ (2,4,6-tri(2-piridil)-1,3,5-triazina), TROLOX (ácido 6-hidroxi-2,5,7,8-tetrametilcroman-2-carboxilico), Folin-Ciocalteau, ácido clorogênico, ácido cafeico, vanilina, ácido ferúlico, ácido para-cumárico, ácido rosmarinico, hesperidina, naringenina, pinobanksina, canferol, canferide e pinostrobina foram adquiridos da Sigma-Aldrich Chemie (Steinheim, Germany).

\section{Extratos etanólicos de própolis bruta (E)}

Um grama de própolis bruta foi extraída com $25 \mathrm{~mL}$ de etanol (P.A., 95\%) sob agitação magnética. Após agitação à temperatura ambiente durante $48 \mathrm{~h}$, estas soluções foram filtradas. O solvente etanólico foi removido sob pressão reduzida produzindo extrato etanólico de própolis identificados como extratos etanólicos de própolis bruta $(\mathrm{E} 1-\mathrm{E} 12)$.

\section{Amostras de própolis comerciais $(\mathbf{C})$}

Os extratos obtidos do comércio estavam na concentração de $30 \%$ $(\mathrm{m} / \mathrm{v})$, conforme especificações que constam no Regulamento Técnico de Identidade e Qualidade do Extrato de Própolis, presente na normativa $\mathrm{n}^{\circ}$. 03, de 19 de Janeiro de 2001, do Ministério da Agricultura e Abastecimento. ${ }^{6}$ Para a realização dos ensaios de determinação do teor em fenólicos e flavonoides totais, capacidade antioxidante, e para a quantificação por CLAE-DAD, todos os extratos de própolis comerciais foram evaporados à pressão reduzida e solubilizados em metanol grau espectroscópico de acordo com a concentração necessária para a realização dos testes. As amostras foram identificadas como extratos comerciais de própolis $(\mathrm{C} 1-\mathrm{C} 12)$.

\section{Determinação do teor de fenólicos totais: método de Folin- Ciocalteau}

Uma alíquota de $50 \mu \mathrm{L}$ da solução do extrato de própolis $(1,00$ $\mathrm{mg} \mathrm{mL}^{-1}$ ) foi misturado com $2,5 \mathrm{~mL}$ de reagente de Folin-Ciocalteu 1:10 e 2,0 mL de solução aquosa $4 \%$ de $\mathrm{Na}_{2} \mathrm{CO}_{3}$. Após 5 minutos de incubação a $50{ }^{\circ} \mathrm{C}$, a absorvância foi medida contra um branco (água ultrapura) a $760 \mathrm{~nm} .{ }^{7}$ A curva de calibração foi preparada com ácido gálico (25 a $\left.1000 \mu \mathrm{g} \mathrm{mL}^{-1} ; \mathrm{Y}=0,12951 X+0,12497 ; \mathrm{R}=0,999\right)$. $\mathrm{O}$ conteúdo de fenólico total foi expresso em mg $\mathrm{E}_{\mathrm{GA}} 100 \mathrm{mg}^{-1} \mathrm{e}$ as análises foram feitas com três repetições.

\section{Determinação do teor de flavonoides totais}

Uma alíquota de $400 \mu \mathrm{L}$ de extrato de própolis $\left(1,00 \mathrm{mg} \mathrm{mL}^{-1}\right)$ e $200 \mu \mathrm{L}$ de solução metanólica de cloreto de alumínio (2\%) foram misturados e diluídos com metanol em balão volumétrico de 10,00 $\mathrm{mL}$. Após 30 minutos a absorvância foi medida a $425 \mathrm{~nm}$ contra um branco (metanol). ${ }^{8}$ A curva de calibração foi preparada com quercetina $\left(1-50 \mu \mathrm{g} \mathrm{mL}^{-1} ; \mathrm{Y}=0,06553+0,04078 X ; \mathrm{R}=0,999\right)$. Os resultados foram expressos em $\mathrm{mg} \mathrm{Q}_{\mathrm{E}} 100 \mathrm{mg}^{-1} \mathrm{e}$ as análises foram feitas com três repetições.

\section{Ensaio de sequestro de radicais DPPH}

Setenta e um microlitros de solução de extrato de própolis (10 - $\left.100 \mu \mathrm{g} \mathrm{mL}^{-1}\right)$ ou do padrão $\left(5-500 \mu \mathrm{mol} \mathrm{L}^{-1}\right)$ foram misturados com $29 \mu \mathrm{L}$ de uma solução $0,3 \mathrm{mmol} \mathrm{L}^{-1}$ de DPPH em metanol. ${ }^{9}$ A mistura foi deixada em repouso durante $30 \mathrm{~min}$ no escuro e, em seguida, a absorvância foi medida a $517 \mathrm{~nm}$ utilizando um leitor de microplacas de ELISA 680 (Bio-Rad, Brasil). Os resultados foram expressos em $\mathrm{CE}_{50}\left(\mu \mathrm{g} \mathrm{mL} \mathrm{m}^{-1}\right)$.

\section{Determinação da atividade antioxidante pela Captura do Radical-Cátion (ABTS ${ }^{+}$)}

Uma alíquota de $50 \mu \mathrm{L}$ do extrato de própolis $\left(1,00 \mathrm{mg} \mathrm{mL}^{-1}\right)$ foi misturado com 5,0 mL do reagente de ABTS e, após 6 minutos, foi realizada a leitura da absorbância a $734 \mathrm{~nm}$ utilizando álcool etílico como o branco. ${ }^{10}$ A curva de calibração foi preparada com Trolox (0,00 - 2,40 $\left.\mathrm{mmol} \mathrm{L}^{-1} ; \mathrm{Y}=-26,37778 \mathrm{X}+0,65164 ; \mathrm{R}=-0,9997\right)$. Os resultados foram expressos em: mmol TE $100 \mathrm{mg}^{-1} \mathrm{e}$ as análises realizadas com três repetições.

\section{Avaliação da atividade antioxidante pelo Método de Redução do Íon Férrico (FRAP)}

Uma alíquota de $0,5 \mathrm{~mL}$ do extrato de própolis $\left(1,00 \mathrm{mg} \mathrm{mL}^{-1}\right)$ foi misturado com 4,5 mL do reagente de FRAP. Após 10 minutos 
de incubação a $37{ }^{\circ} \mathrm{C}$ foi realizada a leitura da absorbância a 593 $\mathrm{nm}$, utilizando água ultrapura como branco. ${ }^{11} \mathrm{~A}$ curva de calibração foi preparada com $\mathrm{FeSO}_{4} \cdot 7 \mathrm{H}_{2} \mathrm{O}(100-1000 \mu \mathrm{M} ; \mathrm{Y}=0,0018 \mathrm{X}+$ 0,00107; $\mathrm{R}=0,99961)$ e os resultados expressos em: mmol Fe(II) $100 \mathrm{mg}^{-1}$. As análises foram realizadas com três repetições.

\section{Análise por Cromatografia Líquida de Alta Eficiência (CLAE- DAD)}

Os perfis cromatográficos dos diferentes extratos de própolis foram determinadas por cromatografia líquida de alta eficiência (CLAE-DAD, Shimadzu), constituído por duas bombas série LC20AT, detector de arranjo de díodos série SPD-M20A e autoinjetor SIL-10A. O controle do equipamento e aquisição dos dados foi feito através do software LCSolution (Shimadzu). As análises foram realizadas em coluna analítica de fase reversa Betasil C-18 (250 x 4,6 $\mathrm{mm}, 5 \mu \mathrm{m}$ de partícula, Thermo) mantida a $30^{\circ} \mathrm{C}$, com taxa de fluxo constante de $1,0 \mathrm{~mL} \mathrm{~min}^{-1}$. A fase móvel foi constituída por uma mistura de água: ácido acético (99:1, solvente A) e metanol (100, solvente B), e um gradiente linear foi realizado começando com $30 \%$ de metanol até $92 \%$ durante $35 \mathrm{~min}$. Todas as soluções de extrato de própolis foram preparadas em metanol grau espectroscópico a 1000 $\mu \mathrm{g} \mathrm{mL} \mathrm{m}^{-1}$ e $20 \mu \mathrm{L}$ foram injetados após serem filtrados através de um filtro de seringa ( $13 \mathrm{~mm}$, porosidade de $0.45 \mu \mathrm{m}$, PVDF, Analítica). A identificação das substâncias presentes nos extratos foi baseada na comparação dos tempos de retenção e suas curvas no UV com os de padrões autênticos, e a quantificação foi realizada usando dados de calibração externo para os mesmos compostos. Cada padrão foi analisado individualmente e o monitoramento dos cromatogramas foi realizado a 280 e $320 \mathrm{~nm}$. Na Tabela 2 estão definidas as equações da reta, a linearidade (R) e a faixa de concentração de cada padrão. Todas as análises foram realizadas em três repetições. Os resultados quantitativos foram expressos em $\mathrm{mg}$ de composto por $100 \mathrm{mg}$ de extrato.

\section{Análise estatística}

Para efetuar a análise de componentes principais (PCA) foi utilizado o software The Unscramble ${ }^{\circledR}$ 10.3. As análises químicas foram realizadas em triplicata para a construção da matriz de dados 24 x 18 (vinte e quatro amostras e dezoito variáveis). Os dados foram autoescalados, centrados na média e foi utilizada a validação cruzada na realização do PCA.

\section{RESULTADOS E DISCUSSÃO}

\section{Conteúdo de fenólicos totais (TP) e conteúdo de flavonoides totais (TF)}

A determinação do teor de fenólicos totais (TP) presentes nas amostras foi feita pelo método espectrofotométrico utilizando o reagente de Folin-Ciocalteau com modificações. ${ }^{7}$ Os resultados estão expressos na Tabela 3. Para os extratos de própolis verde in natura $(\mathrm{E})$ os valores de TP variaram de 1,37 - 14,43 mg E $\mathrm{GA}_{\mathrm{GA}} 100 \mathrm{mg}^{-1}$, enquanto os resultados encontrados nos extratos comerciais (C) apresentaram valores bem superiores, variando de 4,05 - 122,68 mg E $\mathrm{GA}_{\mathrm{GA}} 100 \mathrm{mg}^{-1}$, e os resultados foram expressos por meio de uma curva padrão de ácido gálico $(\mathrm{R}=0,999)$.

O teor de flavonoides (TF) foi determinado pelo método de complexação por cloreto de alumínio que é específico para flavonas e flavonóis. Para a quantificação foi utilizada uma curva analítica de quercetina como padrão $(\mathrm{R}=0,999)$. Os resultados de $\mathrm{TF}$ para os extratos in natura (E) variaram de 0,63 - 9,71 mg E $100 \mathrm{mg}^{-1}$, e foram semelhantes aos encontrados para as tinturas comerciais (C) $0,16-9,13 \mathrm{mg} \mathrm{E}_{\mathrm{Q}} 100 \mathrm{mg}^{-1}$.

Segundo a legislação brasileira vigente os extratos etanólicos de própolis devem conter, no mínimo, $0,25 \%$ de flavonoides e $0,50 \%$ de fenólicos. ${ }^{6}$ Neste trabalho o conteúdo médio de compostos fenólicos e flavonoides foi de $0,89 \mathrm{~g} \%$ e $0,50 \mathrm{~g} \%$ para os extratos de própolis verde coletados (E), e de $8,29 \mathrm{~g} \%$ e $0,50 \mathrm{~g} \%$ para as tinturas comerciais (C), respectivamente, indicando que todas as amostras estavam dentro dos padrões estabelecidos pela legislação quanto a esses dois parâmetros. Como a composição química da própolis é dependente da localização geográfica, a relação entre os teores de fenólicos e flavonoides totais está relacionada com a flora e região da coleta, ${ }^{12}$ bem como podem variar de acordo com o solvente usado na obtenção do extrato de própolis. Assim, os valores superiores de TP obtidos para os extratos comerciais podem estar relacionados à característica mais polar do solvente de extração (etanol 70\%), bem como por serem extratos mais concentrados, na faixa de $30 \%(\mathrm{~m} / \mathrm{v})$, já que para os extratos de própolis verde coletados o solvente extrator foi um pouco menos polar (etanol 95\%) e a concentração dos extratos não ultrapassou $4 \%(\mathrm{~m} / \mathrm{v})$.

Tabela 2. Equações da reta, linearidade (R) e a faixa de concentração de cada padrão

\begin{tabular}{lccc}
\hline Padrões & Equação da reta & $\mathrm{R}$ & Faixa de concentração $\left(\mu \mathrm{g} \mathrm{mL}^{-1}\right)$ \\
\hline Ácido clorogênico & $-90334,46+12886,18 * \mathrm{X}$ & $100-3000$ \\
Ácido cafeico & $153145,60+74,50 * \mathrm{X}$ & 0,99723 & $50-2000$ \\
Ácido para-cumárico & $-337764,37+93909,23 * \mathrm{X}$ & 0,99016 & $50-3000$ \\
Ácido ferúlico & $211001,33+26531,84 * \mathrm{X}$ & 0,99932 & $50-3000$ \\
Ácido rosmarinico & $16627,13+19223,08 * \mathrm{X}$ & 0,99248 & $100-3000$ \\
Hesperidina & $55694,26+20606,75 * \mathrm{X}$ & 0,99923 & $50-1000$ \\
Vanilina & $-134683,19+58078,01 * \mathrm{X}$ & 0,99051 & $100-1000$ \\
Canferol & $-97036,23+45848,86 * \mathrm{X}$ & 0,99817 & $50-1000$ \\
Canferide & $7489+31810,5 * \mathrm{X}$ & 0,99981 & $100-3000$ \\
Naringenina & $-73674,18+32870,47 * \mathrm{X}$ & 0,99987 & $50-1000$ \\
Pinobanksina & $-327949+71201 * \mathrm{X}$ & 0,99979 & $100-2000$ \\
Artepillin C & $-25806,37+9025,74 * \mathrm{X}$ & 0,99705 & $100-3000$ \\
Pinostrombina & $-152223,53+60044,86 * \mathrm{X}$ & 0,99973 & $100-2000$ \\
\hline
\end{tabular}


Tabela 3. Teores de fenólicos (TP), flavonoides (TF) e capacidade antioxidante dos extratos de própolis ${ }^{\mathrm{a}}$

\begin{tabular}{|c|c|c|c|c|c|}
\hline Amostras & $\mathrm{TP}$ & $\mathrm{TF}$ & FRAP & ABTS & $\mathrm{CE}_{50}$ \\
\hline E1 & $8,74 \pm 0,01$ & $7,31 \pm 0,01$ & $260,79 \pm 0,03$ & $98,65 \pm 0,09$ & $42,58 \pm 0,02$ \\
\hline E2 & $7,90 \pm 0,02$ & $5,21 \pm 0,01$ & $487,74 \pm 0,02$ & $99,03 \pm 0,06$ & $36,84 \pm 0,09$ \\
\hline $\mathbf{E 3}$ & $1,37 \pm 0,01$ & $0,63 \pm 0,01$ & $482,18 \pm 0,02$ & $84,86 \pm 0,01$ & $52,76 \pm 0,04$ \\
\hline E4 & $14,43 \pm 0,01$ & $9,71 \pm 0,01$ & $384,96 \pm 0,03$ & $157,62 \pm 0,01$ & $35,61 \pm 0,12$ \\
\hline E5 & $11,88 \pm 0,07$ & $8,73 \pm 0,06$ & $395,79 \pm 0,03$ & $128,89 \pm 0,04$ & $30,65 \pm 0,02$ \\
\hline E6 & $7,15 \pm 0,01$ & $5,59 \pm 0,01$ & $289,41 \pm 0,01$ & $116,64 \pm 0,09$ & $51,27 \pm 0,04$ \\
\hline E7 & $6,86 \pm 0,02$ & $4,96 \pm 0,02$ & $341,63 \pm 0,01$ & $123,73 \pm 0,15$ & $56,71 \pm 0,02$ \\
\hline E8 & $10,57 \pm 0,03$ & $4,56 \pm 0,02$ & $381,35 \pm 0,01$ & $136,36 \pm 0,05$ & $50,33 \pm 0,05$ \\
\hline E9 & $9,16 \pm 0,02$ & $1,45 \pm 0,02$ & $291,91 \pm 0,01$ & $149,95 \pm 0,02$ & $35,79 \pm 0,05$ \\
\hline E10 & $8,46 \pm 0,01$ & $2,28 \pm 0,01$ & $283,29 \pm 0,02$ & $110,14 \pm 0,02$ & $31,95 \pm 0,01$ \\
\hline E11 & $9,89 \pm 0,02$ & $3,40 \pm 0,01$ & $332,74 \pm 0,01$ & $112,43 \pm 0,02$ & $39,75 \pm 0,06$ \\
\hline E12 & $10,47 \pm 0,02$ & $5,87 \pm 0,02$ & $608,85 \pm 0,03$ & $142,68 \pm 0,04$ & $32,98 \pm 0,05$ \\
\hline Média & 8,90 & 4,97 & 378,38 & 121,74 & 41,43 \\
\hline C1 & $121,53 \pm 0,05$ & $6,13 \pm 0,03$ & $457,46 \pm 0,05$ & $214,86 \pm 0,01$ & $34,35 \pm 0,05$ \\
\hline $\mathrm{C2}$ & $97,56 \pm 0,03$ & $0,16 \pm 0,05$ & $65,52 \pm 0,03$ & $117,03 \pm 0,01$ & $100,32 \pm 0,03$ \\
\hline $\mathrm{C3}$ & $93,98 \pm 0,02$ & $2,62 \pm 0,03$ & $221,63 \pm 0,02$ & $223,67 \pm 0,01$ & $32,12 \pm 0,02$ \\
\hline $\mathrm{C} 4$ & $5,46 \pm 0,01$ & $9,13 \pm 0,02$ & $657,74 \pm 0,01$ & $246,83 \pm 0,05$ & $19,36 \pm 0,01$ \\
\hline C5 & $92,79 \pm 0,03$ & $4,49 \pm 0,05$ & $362,46 \pm 0,06$ & $167,76 \pm 0,01$ & $49,31 \pm 0,02$ \\
\hline C6 & $4,05 \pm 0,01$ & $6,46 \pm 0,04$ & $308,85 \pm 0,01$ & $183,84 \pm 0,03$ & $46,23 \pm 0,01$ \\
\hline C7 & $122,68 \pm 0,05$ & $4,89 \pm 0,06$ & $233,02 \pm 0,05$ & $174,66 \pm 0,06$ & $33,58 \pm 0,05$ \\
\hline C8 & $86,57 \pm 0,02$ & $5,27 \pm 0,06$ & $371,07 \pm 0,04$ & $161,25 \pm 0,01$ & $54,13 \pm 0,04$ \\
\hline C9 & $78,66 \pm 0,02$ & $4,24 \pm 0,03$ & $427,46 \pm 0,04$ & $154,17 \pm 0,10$ & $30,77 \pm 0,19$ \\
\hline C10 & $87,38 \pm 0,06$ & $4,15 \pm 0,07$ & $283,85 \pm 0,05$ & $166,42 \pm 0,15$ & $48,07 \pm 0,01$ \\
\hline C11 & $92,93 \pm 0,05$ & $5,25 \pm 0,02$ & $412,46 \pm 0,06$ & $193,99 \pm 0,20$ & $31,98 \pm 0,02$ \\
\hline $\mathrm{C12}$ & $111,90 \pm 0,04$ & $6,89 \pm 0,01$ & $411,35 \pm 0,04$ & $212,94 \pm 0,10$ & $27,11 \pm 0,06$ \\
\hline Média & 82,95 & 4,97 & 351,07 & 184,78 & 42,27 \\
\hline
\end{tabular}

${ }^{a}$ Testes realizados com três repetições. Valores expressos em: TP $\left(\mathrm{mgE}_{\mathrm{AG}} 100 \mathrm{mg}^{-1}, \mathrm{TF}\left(\mathrm{mgE}_{\mathrm{Q}} 100 \mathrm{mg}^{-1}\right), \mathrm{FRAP}\left(\mathrm{mmol} \mathrm{Fe}(\mathrm{II}) 100 \mathrm{mg}^{-1}\right), \mathrm{ABTS}(\mathrm{mmolTE} 100\right.$ $\left.\mathrm{mg}^{-1}\right)$ e $\mathrm{CE}_{50}\left(\mu \mathrm{gLL}^{-1}\right)$ de extrato.

Atualmente existe um grande interesse no estudo das substâncias com propriedades antioxidantes, devido, principalmente, às descobertas sobre o efeito dos radicais livres no organismo. Na Tabela 3 estão apresentados os resultados da capacidade antioxidante dos extratos de própolis bruta e comercial realizados por três diferentes métodos: DPPH, ABTS e FRAP, que são amplamente utilizados devido à simplicidade, rapidez, sensibilidade e reprodutibilidade.

Os resultados obtidos para ambos os extratos (E e C) neste trabalho, utilizando o ensaio com DPPH, foram expressos como $\mathrm{CE}_{50} \mathrm{e}$ variaram entre 19,36 a $100,32 \mu \mathrm{g} \mathrm{mL}^{-1}$. Entre as amostras, observa-se que existe correlação significativa entre a quantidade de TF e a capacidade antioxidante (DPPH), como no caso da amostra C4, que apresentou teor mais elevado de $\mathrm{TF}$ e o melhor valor de $\mathrm{CE}_{50}$. Este fato não pode ser atribuído ao conteúdo de TP, visto que não se observou uma correlação significativa com a capacidade antioxidante, como observado na amostra C7. Outro fator que também poderia ter contribuído para a menor capacidade antioxidante das amostras decorreu do fato das mesmas apresentarem-se ricas em cera e substâncias não polares, como foi observado na amostra E3. Estes resultados indicam que a concentração total de compostos fenólicos ou flavonoides não é o único fator responsável pelas propriedades antioxidantes. A natureza química das substâncias fenólicas e, talvez, a presença de outros derivados contribuam para a capacidade antioxidante total dos extratos. A forte capacidade antioxidante na própolis pode vir dos flavonoides, como canferol e canferide, porém, os fenólicos podem participar juntos com os flavonoides na determinação dessas atividades antioxidantes.

De maneira geral, as amostras C4 e E12 apresentaram os melhores resultados de atividade antirradicalar. Os valores de capacidade antioxidante em FRAP, ABTS e DPPH foram de 657,74 e 608,85 mmol Fe(II) $100 \mathrm{mg}^{-1} ; 246,83$ e 142,68 mmol TE $100 \mathrm{mg}^{-1} ; 19,36 \mathrm{a}$ $32,98 \mathrm{mg} \mathrm{mL}^{-1}$, respectivamente.

Apesar dos extratos comerciais terem apresentado valores mais elevados de TP quando comparados aos extratos in natura, esse resultado não refletiu diretamente em um aumento na capacidade antioxidante. Para auxiliar no entendimento e visualização das potenciais correlações entre estes diferentes parâmetros foi desenvolvida uma matriz de correlação (Tabela 4). Os resultados demonstraram uma correlação positiva com aumento do teor de flavonoides totais (TF) influenciando a capacidade antioxidante total. Essa matriz revelou alta correlação entre o teor de flavonoides (TF) e o potencial antioxidante realizado pelos três métodos: FRAP $(r=0,87)$, DPPH $(r=$ $-0,74)$ e DPPH $(r=0,74)$. Em relação aos fenólicos (TP) não foram encontradas correlações significativas. Uma análise mais detalhada da Tabela 3 mostrou que a capacidade antioxidante da própolis não pode ser relacionada à concentração de compostos fenólicos, uma 
vez que a amostra E12 teve o dobro da concentração de fenólicos quando comparada a $\mathrm{C} 4$, e esta teve maior atividade antirradicalar.

Tabela 4. Matriz de correlação entre as variáveis analisadas para os extratos de própolis

\begin{tabular}{lccccc}
\hline & TP & TF & FRAP & ABTS & DPPH \\
\hline TP & 1 & & & & \\
\hline TF & $-0,45^{*}$ & 1 & & & \\
\hline FRAP & $-0,39^{*}$ & $0,87^{*}$ & 1 & & \\
\hline ABTS & $-0,25^{*}$ & $0,71^{*}$ & $0,66^{*}$ & 1 & \\
\hline DPPH & $0,13^{*}$ & $-0,74^{*}$ & $-0,73^{*}$ & $-0,80^{*}$ & 1 \\
\hline
\end{tabular}

*Significativo ao nível de $\rho \leq 0.05$.

Atualmente, a própolis tem sido usada como uma terapia alternativa em virtude de seus benefícios medicinais e até mesmo na forma de alimento funcional. A sua comercialização ocorre na maior parte na forma de extratos alcoólicos ou hidroalcoólicos. ${ }^{13}$

$\mathrm{O}$ aumento do consumo dessas tinturas leva a uma preocupação sobre a necessidade de se conhecer a sua composição química a fim de tentar estabelecer uma correlação entre as substâncias identificadas e as suas propriedades biológicas. Assim, é importante se preocupar para que durante o processo de preparação do extrato não se deteriore o produto, e dessa forma seja assegurada a qualidade dos extratos para aplicação terapêutica.

Este trabalho apresentou uma comparação entre composição química e a atividade antioxidante de amostras de extratos de própolis verde in natura e tinturas que são comercializadas em diferentes regiões do sudeste brasileiro, a fim qualificar o seu perfil químico.

De acordo com estes dados, as tinturas de própolis etanólicas encontradas no comércio apresentaram um bom teor de fenólicos e flavonoides. Além disso, foi possível determinar a capacidade antioxidante e estabelecer a composição química por CLAE-DAD que foi semelhante aos das amostras de própolis in natura.

\section{Identificação dos constituintes fenolicos usando CLAE-DAD}

Os dois grupos de própolis avaliadas neste trabalho apresentaram composição qualitativa semelhantes por CLAE-DAD, e treze subtâncias foram identificadas. A caracterização do perfil químico dos diferentes extratos de própolis foi realizada por comparação dos tempos de retenção $\left(t_{R}\right)$ e absorções máximas no espectro de UV com padrões autênticos. A análise revelou a presença de derivados de ácidos fenólicos, aldeído, flavonoides glicosilados e agliconas. Entre os extratos analisados foi possível identificar e quantificar vanilina, hesperidina, naringenina, pinostrombina, pinobanksina, canferol e canferide, além dos ácidos clorogênico, caféico, para-cumárico, ferúlico, rosmarínico e artepilin $\mathrm{C}$, em ambos os extratos, própolis in natura (E) e tinturas comerciais (C). Cabe destacar a presença em todos os extratos de artepillin C (ácido 3,5-diprenil-4-hidroxicinâmico), substância considerada um biomarcador da própolis verde comum na região sudeste. ${ }^{14}$

Na Figura 1 estão apresentados os cromatogramas das amostras E12 e C4. Nestas duas amostras foram identificadas as substâncias: ácido clorogênico (CLA), ácido caféico (CA), ácido ferúlico (FA), ácido para-cumárico ( $p$-CA), ácido rosmarínico (RA), artepillin-C $(\mathrm{ArtC})$, hesperidina $(\mathrm{H})$; naringenina $(\mathrm{N})$, pinobanksina $(\mathrm{PK})$, canferol (K), canferide (C) e pinostronbina (PT). Os ácidos para-cumárico, clorogênico e caféico, assim como o artepillin C são citados na literatura como constituintes de própolis verde. ${ }^{15}$

Os ácidos cafeico, para-cumárico e artepillin C, e os flavonoides galangina, pinocembrina e pinobanksina também foram identificados nos extratos de própolis verde da África do Sul, ${ }^{15}$ sendo semelhante ao encontrado nos extratos E e C. Da mesma forma, em própolis verde chinesa, também foram identificados os ácidos caféico, para-cumárico, ferúlico, além de pinobanksina, naringenina, canferol, pinocembrina e galangina. ${ }^{16}$

A avaliação da capacidade antioxidante das substâncias identificadas nos extratos foi determinada individualmente com a finalidade de se tentar estabelecer uma possível correlação entre as substâncias que
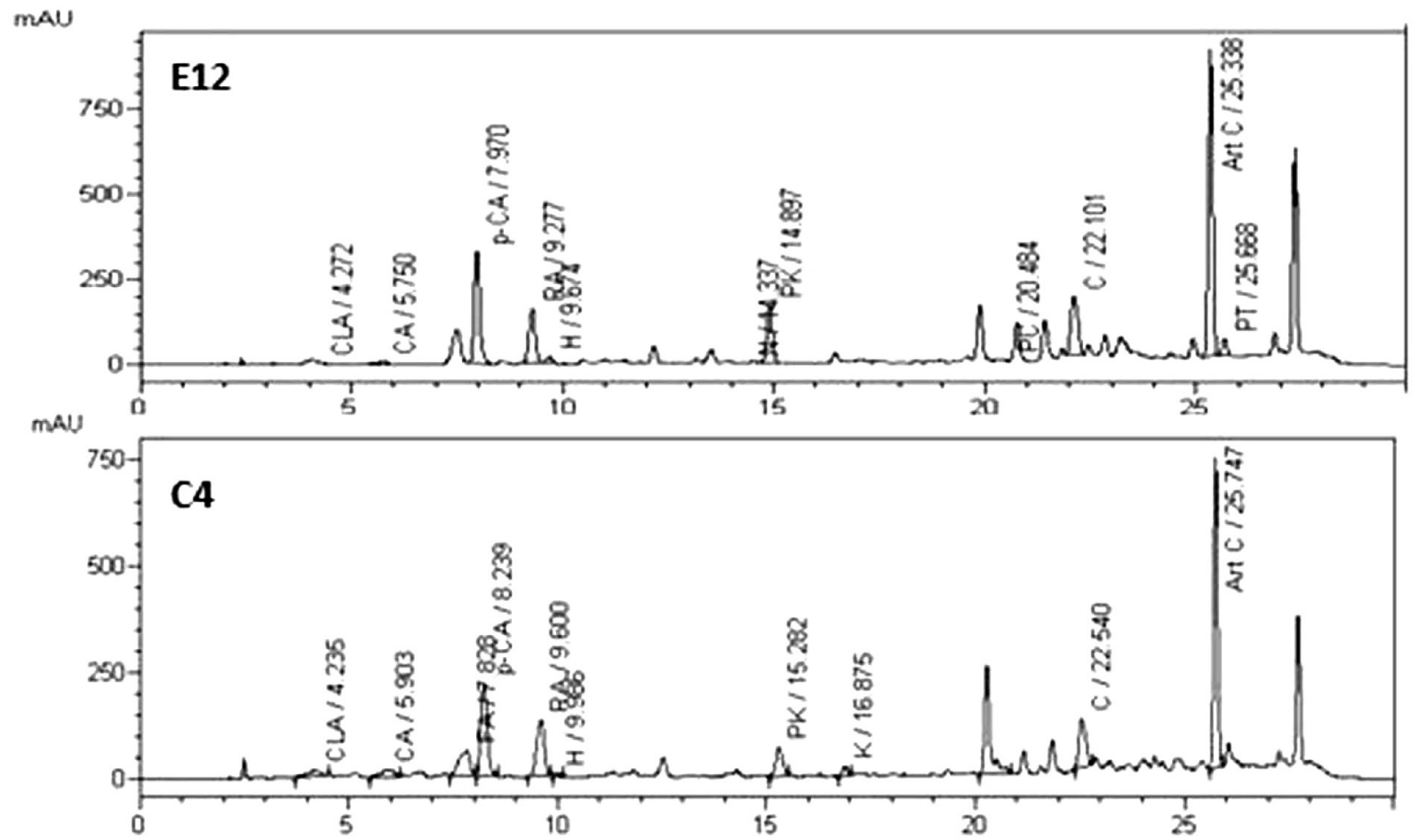

Figura 1. Cromatogramas obtidos em coluna C-18 (25 cm x 4,6 mmx 5 بm), fluxo de 1,0 $\mathrm{mL} \mathrm{min}^{-1}$, fase móvel: água: ácido acético (99:1, solvente A) e metanol (100, solvente B), DAD a $280 \mathrm{~nm}$ para as amostras E12 e C4 
foram identificadas e quantificadas nos extratos de própolis e a sua propriedade antirradicalar. Na Tabela 5 são apresentados os tempos de retenção, as curvas no UV e a capacidade antioxidante $\left(\mathrm{CE}_{50}\right)$ das substâncias padrões que foram identificadas nos extratos. ${ }^{17}$

$\mathrm{Na}$ Tabela 6 estão apresentados os resultados de quantificação

Tabela 5. Tempo de retenção (min), máximo de absorção (nm) e capacidade antioxidante $\left(\mu \mathrm{g} \mathrm{mL}^{-1}\right)$ das substâncias fenólicas utilizados como padrões

\begin{tabular}{lccc}
\hline Padrões & $\mathrm{t}_{\mathrm{R}}(\min )$ & $\lambda_{\max }(\mathrm{nm})$ & $\mathrm{CE}_{50}\left(\mu \mathrm{g} \mathrm{mL}^{-1}\right)$ \\
\hline CLA - Ácido clorogênico & 4,17 & 327 & 5,85 \\
CA - Ácido cafeico & 5,74 & 325 & 9,30 \\
pCA - Ácido para-cumárico & 8,46 & 310 & 39,31 \\
FA - Ácido ferúlico & 8,19 & 240,322 & 24,71 \\
RA - Ácido rosmarinico & 9,16 & 330 & $>20$ \\
H- Hesperidina & 9,56 & 283 & 106,55 \\
V- Vanilina & 7,03 & 270,309 & 486,01 \\
K- Canferol & 11,02 & 267,365 & 6,10 \\
C- Canferide & 22,54 & 266,364 & $>10$ \\
N- Naringenina & 14,55 & 288 & $>1000$ \\
PK- Pinobanksina & 14,90 & 291 & $>500$ \\
Art C- Artepillin C & 25,60 & 314 & 7,38 \\
PT- Pinostrombina & 25,65 & 289 & $>500$ \\
\hline
\end{tabular}

*Referência 17 das substâncias identificados nos extratos, expressos em mg por 100 $\mathrm{mg}$ de extrato. Dos resultados obtidos para as amostras C4 e E12 foi possível observar a presença dos ácidos clorogênico $(0,21$ e 3,32 mg $\left.100 \mathrm{mg}^{-1}\right)$, ferúlico $\left(0,93\right.$ e $\left.3,26 \mathrm{mg} 100 \mathrm{mg}^{-1}\right)$ e rosmarínico $(2,53 \mathrm{e}$

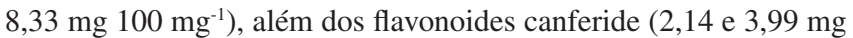

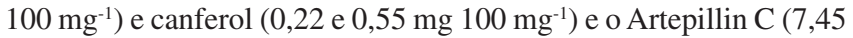

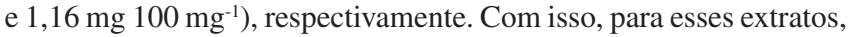
foi possível estabelecer uma relação entre as substâncias identificadas com os melhores resultados da capacidade antioxidante (Tabela 2). Todas essas substâncias identificadas nesses extratos apresentam $\mathrm{CE}_{50}$ inferiores a $24,00 \mu \mathrm{g} \mathrm{mL}^{-1}$, o que pode corroborar para os melhores resultados de capacidade antirradicalar dessas amostras.

Um dos objetivos na utilização da análise multivariada foi reduzir a representação dimensional dos dados e, assim, organizá-los em uma estrutura que facilite a visualização de todo o conjunto de dados. ${ }^{18}$ Para melhor comparar os extratos de própolis quanto às variáveis consideradas e correlacionar esses dados com o tipo de substância e atividade antioxidante encontradas foi aplicada a Análise das Componentes Principais (PCA).

\section{Análise quimiométrica}

Os métodos estatísticos multivariados consideram as amostras e as variáveis em seu conjunto, permitindo extrair informações complementares que a análise univariada não consegue discriminar. O método de análise exploratória de dados foi feito pela Análise de Componentes Principais (PCA), que é geralmente uma ferramenta eficiente para reduzir a dimensionalidade dos dados, além de fornecer

Tabela 6. Conteúdo das substâncias identificadas nas amostras de extratos de própolis (mg $100 \mathrm{mg}^{-1}$ de extrato)

\begin{tabular}{|c|c|c|c|c|c|c|c|c|c|c|c|c|c|}
\hline & CLA & $\mathrm{CA}$ & $\mathbf{V}$ & FA & $p-\mathrm{CA}$ & $\mathbf{R A}$ & $\mathbf{H}$ & $\mathbf{N}$ & PK & $\mathbf{K}$ & Art C & C & PT \\
\hline E1 & $0,24 \pm 0,01$ & $0,05 \pm 0,02$ & & $0,35 \pm 0,05$ & $0,61 \pm 0,01$ & $0,45 \pm 0,02$ & $0,05 \pm 0,01$ & & $0,62 \pm 0,01$ & $0,13 \pm 0,01$ & $0,18 \pm 0,02$ & $1,43 \pm 0,01$ & \\
\hline E2 & $0,22 \pm 0,02$ & $0,08 \pm 0,01$ & & $0,71 \pm 0,04$ & $1,13 \pm 0,01$ & $1,93 \pm 0,03$ & $0,17 \pm 0,02$ & $0,36 \pm 0,02$ & $0,48 \pm 0,03$ & $0,23 \pm 0,02$ & $1,57 \pm 0,05$ & $2,64 \pm 0,01$ & $0,21 \pm 0,02$ \\
\hline $\mathbf{E 3}$ & $0,10 \pm 0,01$ & & & $0,03 \pm 0,01$ & $0,08 \pm 0,02$ & $0,10 \pm 0,00$ & $0,01 \pm 0,01$ & $0,03 \pm 0,01$ & $0,13 \pm 0,02$ & $0,04 \pm 0,01$ & $0,06 \pm 0,01$ & $0,19 \pm 0,01$ & \\
\hline E4 & $0,24 \pm 0,03$ & $0,46 \pm 0,05$ & & $0,92 \pm 0,02$ & $1,09 \pm 0,09$ & $1,35 \pm 0,17$ & $0,06 \pm 0,02$ & $0,10 \pm 0,02$ & $1,12 \pm 0,10$ & $0,16 \pm 0,01$ & $0,35 \pm 0,01$ & & $1,97 \pm 0,11$ \\
\hline E5 & $0,24 \pm 0,02$ & $0,17 \pm 0,06$ & & $0,36 \pm 0,02$ & $1,37 \pm 0,10$ & $0,36 \pm 0,00$ & $0,05 \pm 0,02$ & $0,08 \pm 0,01$ & $1,01 \pm 0,04$ & $0,25 \pm 0,02$ & $1,53 \pm 0,07$ & $3,27 \pm 0,13$ & \\
\hline E6 & $0,21 \pm 0,01$ & $0,28 \pm 0,04$ & & $0,60 \pm 0,05$ & $0,80 \pm 0,05$ & $0,60 \pm 0,01$ & $0,04 \pm 0,02$ & $0,10 \pm 0,02$ & $0,85 \pm 0,05$ & $0,18 \pm 0,01$ & $0,65 \pm 0,01$ & $2,53 \pm 0,20$ & \\
\hline E7 & $0,22 \pm 0,02$ & $0,02 \pm 0,01$ & & $0,07 \pm 0,01$ & $0,45 \pm 0,03$ & $0,32 \pm 0,00$ & $0,04 \pm 0,01$ & $0,11 \pm 0,03$ & $0,31 \pm 0,01$ & $0,11 \pm 0,01$ & $0,10 \pm 0,01$ & $0,66 \pm 0,06$ & \\
\hline E8 & $0,18 \pm 0,01$ & $0,05 \pm 0,02$ & $0,14 \pm 0,02$ & $0,04 \pm 0,01$ & $0,48 \pm 0,02$ & $0,54 \pm 0,00$ & $0,04 \pm 0,02$ & $0,07 \pm 0,01$ & $0,45 \pm 0,03$ & & $0,24 \pm 0,07$ & $0,86 \pm 0,05$ & \\
\hline E9 & & & $0,63 \pm 0,03$ & $0,71 \pm 0,03$ & $0,31 \pm 0,02$ & $0,05 \pm 0,00$ & & $0,15 \pm 0,03$ & $0,77 \pm 0,07$ & & $0,11 \pm 0,01$ & & \\
\hline E10 & $0,17 \pm 0,03$ & $0,20 \pm 0,02$ & & $0,33 \pm 0,02$ & $1,00 \pm 0,01$ & $0,82 \pm 0,00$ & $0,04 \pm 0,01$ & $0,08 \pm 0,02$ & $1,00 \pm 0,03$ & & $1,07 \pm 0,02$ & $2,18 \pm 0,08$ & \\
\hline E11 & $0,20 \pm 0,03$ & $0,13 \pm 0,01$ & $0,35 \pm 0,03$ & $0,28 \pm 0,01$ & $0,66 \pm 0,01$ & $0,45 \pm 0,00$ & $0,03 \pm 0,01$ & $0,10 \pm 0,02$ & $0,76 \pm 0,05$ & & $0,28 \pm 0,01$ & $3,97 \pm 0,10$ & \\
\hline E12 & $0,21 \pm 0,02$ & $0,13 \pm 0,02$ & & $0,93 \pm 0,06$ & $1,03 \pm 0,03$ & $2,53 \pm 0,00$ & $0,15 \pm 0,02$ & $0,08 \pm 0,01$ & $0,79 \pm 0,07$ & $0,22 \pm 0,02$ & $1,16 \pm 0,35$ & $2,14 \pm 0,01$ & \\
\hline $\mathrm{C} 1$ & $2,13 \pm 0,02$ & $0,19 \pm 0,04$ & & $1,46 \pm 0,10$ & $1,28 \pm 0,06$ & $0,45 \pm 0,00$ & $0,71 \pm 0,01$ & & $0,12 \pm 0,02$ & $0,41 \pm 0,03$ & $2,21 \pm 0,02$ & $1,81 \pm 0,07$ & $0,38 \pm 0,04$ \\
\hline $\mathrm{C} 2$ & $0,84 \pm 0,05$ & $0,07 \pm 0,02$ & & $0,43 \pm 0,04$ & $0,55 \pm 0,05$ & $1,56 \pm 0,00$ & $0,02 \pm 0,01$ & & $0,33 \pm 0,02$ & $0,11 \pm 0,01$ & $2,28 \pm 0,01$ & $0,42 \pm 0,02$ & \\
\hline $\mathrm{C3}$ & $0,62 \pm 0,04$ & $0,05 \pm 0,01$ & $0,54 \pm 0,02$ & $0,24 \pm 0,03$ & $0,59 \pm 0,06$ & $0,70 \pm 0,05$ & & & $0,68 \pm 0,05$ & & $0,70 \pm 0,50$ & $0,68 \pm 0,03$ & $0,23 \pm 0,01$ \\
\hline $\mathrm{C} 4$ & $3,32 \pm 0,15$ & $0,55 \pm 0,02$ & & $3,26 \pm 0,15$ & $2,93 \pm 0,14$ & $8,33 \pm 0,20$ & $0,22 \pm 0,02$ & & $1,52 \pm 0,03$ & $0,55 \pm 0,02$ & $7,45 \pm 0,14$ & $3,99 \pm 0,23$ & $0,89 \pm 0,02$ \\
\hline C5 & $1,30 \pm 0,10$ & $0,10 \pm 0,02$ & & $0,65 \pm 0,05$ & $1,51 \pm 0,13$ & $2,17 \pm 0,18$ & $0,14 \pm 0,01$ & & $1,11 \pm 0,10$ & $0,37 \pm 0,03$ & $1,66 \pm 0,00$ & $2,10 \pm 0,10$ & $0,41 \pm 0,03$ \\
\hline C6 & $1,79 \pm 0,09$ & $0,29 \pm 0,05$ & & $1,06 \pm 0,05$ & $1,69 \pm 0,19$ & $2,97 \pm 0,20$ & $0,16 \pm 0,02$ & & $1,34 \pm 0,16$ & $0,41 \pm 0,05$ & $0,46 \pm 0,01$ & $2,66 \pm 0,20$ & $0,43 \pm 0,02$ \\
\hline $\mathrm{C} 7$ & $2,07 \pm 0,06$ & $0,09 \pm 0,02$ & & $0,70 \pm 0,03$ & $1,37 \pm 0,12$ & $3,62 \pm 0,16$ & $0,05 \pm 0,01$ & & $0,87 \pm 0,07$ & $0,33 \pm 0,02$ & $1,83 \pm 0,00$ & $1,32 \pm 0,08$ & $0,42 \pm 0,01$ \\
\hline $\mathrm{C8}$ & $1,16 \pm 0,06$ & $0,11 \pm 0,03$ & & $0,62 \pm 0,03$ & $1,63 \pm 0,11$ & $1,73 \pm 0,08$ & $0,12 \pm 0,05$ & & $1,29 \pm 0,09$ & $0,42 \pm 0,02$ & $1,20 \pm 0,01$ & $2,84 \pm 0,09$ & $0,46 \pm 0,04$ \\
\hline C9 & & $0,03 \pm 0,01$ & & $0,42 \pm 0,02$ & $1,43 \pm 0,10$ & $1,48 \pm 0,09$ & $0,12 \pm 0,03$ & $0,24 \pm 0,03$ & $1,17 \pm 0,07$ & $0,39 \pm 0,03$ & $2,11 \pm 0,01$ & & $0,50 \pm 0,03$ \\
\hline C10 & $1,01 \pm 0,07$ & $1,51 \pm 0,12$ & $0,43 \pm 0,02$ & $0,15 \pm 0,02$ & $1,27 \pm 0,13$ & $1,18 \pm 0,05$ & $0,22 \pm 0,04$ & & $1,22 \pm 0,02$ & $0,39 \pm 0,08$ & $0,77 \pm 0,01$ & $1,93 \pm 0,10$ & $0,45 \pm 0,06$ \\
\hline C11 & $1,36 \pm 0,06$ & $0,04 \pm 0,01$ & & $0,79 \pm 0,08$ & $1,82 \pm 0,20$ & $2,26 \pm 0,10$ & $0,23 \pm 0,02$ & $0,32 \pm 0,02$ & $1,65 \pm 0,06$ & $0,45 \pm 0,05$ & $0,95 \pm 0,01$ & $3,17 \pm 0,15$ & $0,42 \pm 0,03$ \\
\hline $\mathrm{C} 12$ & $2,21 \pm 0,05$ & $0,11 \pm 0,02$ & & $1,06 \pm 0,05$ & $1,92 \pm 0,10$ & $3,76 \pm 0,18$ & $0,07 \pm 0,01$ & $0,31 \pm 0,01$ & $1,01 \pm 0,05$ & $0,44 \pm 0,04$ & $3,50 \pm 0,06$ & $3,24 \pm 0,18$ & $0,39 \pm 0,02$ \\
\hline
\end{tabular}

CLA- ácido clorogênico; CA- ácido cafeico; V- vanilina; FA - ácido ferúlico; p-CA - ácido para-cumárico; RA- ácido rosmarinico; H- hesperidina; N-naringenina; PK- pinobanksina; K-camferol; Art C - Artepillin-C; C-canferide, PT- pinostrombina. 
uma melhor visualização dos agrupamentos entre as amostras. É um método exploratório porque auxilia na elaboração de hipóteses a partir dos dados coletados. A análise de PCA foi realizada considerando os doze extratos etanólicos in natura de própolis (E) e as doze tinturas comerciais $(\mathrm{C})$.

A técnica de análise multivariada foi utilizada visando resumir em poucas e importantes dimensões a maior parte da variabilidade da matriz para a obtenção de relações entre os dados obtidos, extraindo desta maneira a informação mais relevante para o resultado.

A partir dos dados de variância determinou-se o número de PCs. Nessa análise foram escolhidos os dois primeiros PCs. As Tabelas 3 e 6 apresentam os conjuntos de dados experimentais utilizados para análise exploratória PCA, que permitiu a construção de um gráfico de scores (Figura 2). Verificou-se que as componentes PC1 e PC2 descreveram $96 \%$ da variação total dos dados e forneceram informações discriminatórias das amostras.

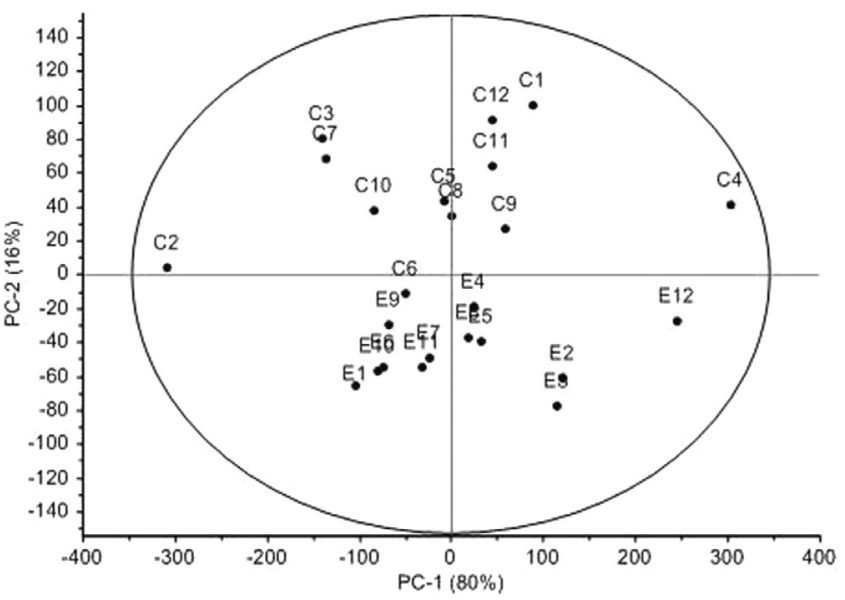

Figura 2. Gráfico dos scores de PC-1 x PC-2

A primeira componente principal (PC1) descreve $80 \%$ da variação total e a segunda (PC2) 16\%. A Figura 3 apresenta o gráfico de scores e observam-se dois agrupamentos. As amostras comerciais (C) estão localizadas na parte superior, enquanto as amostras (E) encontram-se na parte inferior do gráfico. Apesar dos extratos in natura (E) e das tinturas comerciais (C) apresentarem os parâmetros químicos semelhantes, foi possível observar a discriminação em dois grupos distintos no gráfico de scores. Já o gráfico de loadings na Figura 3 mostrou a influência das variáveis sobre as amostras.

Atráves da análise do gráfico de loadings foi possível identificar

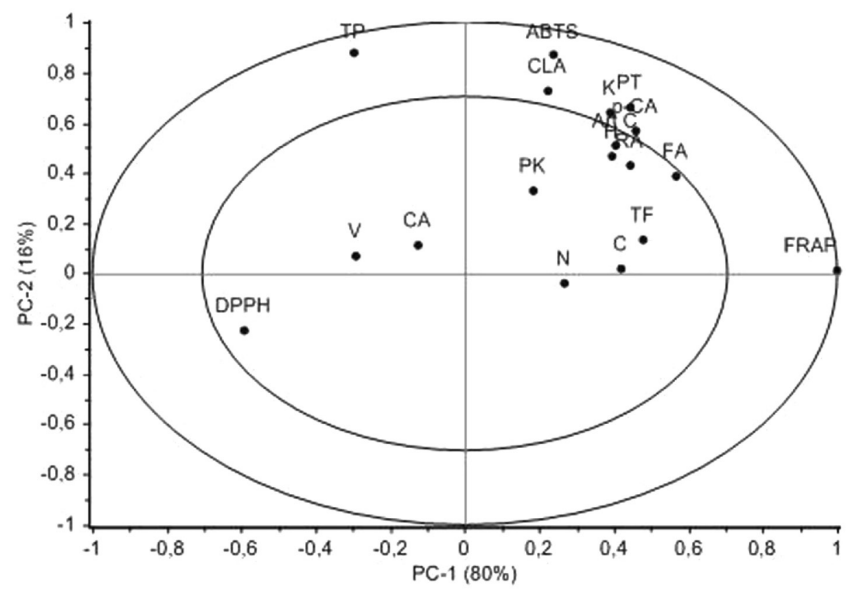

Figura 3. Gráfico dos loadings as váriaveis que contribuiram para a discriminação entre as amostras in natura (E) e comerciais (C). As substâncias hesperidina, canferol e os ácidos para-cumárico, clorogênico, ferúlico e rosmarínico foram importantes para a construção do gráfico, assim como os dados oriundos das análises de ABTS, FRAP e TP. Deve-se destacar a importante contribuição das substâncias quantificadas, que mesmo sendo identificadas em ambos os tipos de extratos em concentrações semelhantes, exerceram um poder discriminante das amostras avaliadas.

\section{CONCLUSÃO}

Neste estudo, determinou-se que a quantidade total de compostos fenólicos, flavonoides e a capacidade antioxidante foram parâmetros importantes para avaliar e comparar a qualidade de extratos in natura e tinturas comerciais a partir da própolis verde brasileira. A atividade antioxidante foi comprovada por dois mecanismos (sequestrante de radical livre e oxi-redução). Os resultados da determinação de teores de fenólicos e flavonoides, o potencial antioxidante (DPPH, ABTS e FRAP), assim como a identificação e quantificação de treze substâncias foram usados para estabelecer uma comparação e indicar uma diferença entre os extratos etanólicos de amostras in natura e das tinturas comerciais. As amostras diferiram entre si em relação aos conteúdos de fenólicos e flavonoides totais, bem como na quantificação das susbtâncias analisadas por CLAE-DAD. Essas variações entre as amostras já eram esperadas, considerando-se que a diferença entre os extratos de própolis pode está relacionada com a flora e região da coleta, bem como pode variar de acordo com o solvente de extração usado na obtenção do extrato de própolis. Os resultados obtidos neste estudo confirmam a influência da origem da matéria-prima na composição química e nas características dos extratos de própolis. Apesar desta diversidade química, todos os extratos de própolis mostraram uma significativa capacidade antioxidante e, na maioria dos casos, pode-se sugerir que as substâncias responsáveis foram os constituintes fenólicos presentes na própolis. Finalmente, deve-se observar que a análise exploratória dos dados permitiu a obtenção de informações rápidas e eficientes sobre a similaridade entre as amostras de própolis pela visualização gráfica, indicando assim a sua procedência in natura ou comercial.

\section{MATERIAL SUPLEMENTAR}

O material suplementar está disponível em http://quimicanova. sbq.org.br, na forma de arquivo PDF com acesso livre, e contém os cromatogramas dos extratos in natura e tinturas comerciais.

\section{AGRADECIMENTOS}

Os autores agradecem as instituições de fomento à pesquisa CNPq, FAPERJ e CAPES pelo auxílio financeiro e pela bolsa de estudo.

\section{REFERÊNCIAS}

1. Park, Y. K.; Alencar, S. M.; Aguiar, C. L.; J. Agric. Food Chem. 2002, 50, 2502; Silva-Carvalho, R.; Baltazar, F.; Almeida-Aguiar, C.; Evidence-Based Complementary Altern. Med. 2015, ID 206439.

2. Toreti, V. C.; Sato, H. H.; Pastore, G. M.; Park, Y. K.; Evidence-Based Complementary Altern. Med. 2013, ID 697390

3. Huang, S.; Zhang, C.; Wang, K.; Li, G. Q.; Hu, F. Molecules 2014, 19, 19610.

4. Sforcin, J. M.; Bankova, V.; J. Ethnopharmacol. 2011, 133, 253.

5. SEBRAE. Boletim: O Mercado da própolis. http://www.sebrae2014. com.br - acessado em 11/12/14. 
6. http://www.apacame.org.br/mensagemdoce/60/normas.htm, acessada em Maio 2016.

7. Papotti, G.; Bertelli, D.; Rossi, M. C.; Plessi, M.; Int. J. Food Sci. Technol. 2010, 45, 1610

8. Mihai, C. M.; Mărghitaş, L. A.; Dezmirean, D. S.; Chirilă, F.; Moritz, R. F.; Schlüns, H.; J. Invertebr. Pathol. 2012, 110, 678

9. Ahn M. R.; Kumazawa S.; Usui Y.; Nakamura J.; Matsuka M.; Zhu F.; Nakayama T.; Food Chem. 2007, 101, 1383; Salgueiro, F. B.; Lira, A. F.; Rumjanek, V. M.; Castro, R. N.; Quim. Nova 2014, 5, 821.

10. Rufino, M. S. M; Alves, R. E.; Brito, E. S.; Morais, S. M.; Sampaio, C. G.; Perez-Jimenez, J.; Saura-Calixto, F. D.; EMBRAPA Comunicado Técnico 128, Fortaleza - CE, 2007.

11. Bertoncelj, J.; Dobersek, U.; Jamnik, M.; Golob, T.; Bertoncelj, J.; Dobersek, U.; Jamnik, M.; Golob, T.; Food Chem. 2007, 105, 822; Sant'ana, L. D'O.; Sousa, J. L. M.; Salgueiro, F. B.; Lorenzon, M. C. A.; Castro, R. N.; J. Food Sci. 2012, 71, 135.

12. Bankova V.; J. Ethnopharmacol. 2005, 100, 114.
13. Lustosa, S. R.; Galindo, A. B.; Nunes, L. C. C.; Randau, K. P.; Neto, P. J. R.; Rev. Bras. Farmacogn. 2008, 18, 447.

14. Nobushi, Y.; Oikawa, N.; Okazaki, Y.; Tsutsumi, S.; Kun Park, Y.; Kurokawa, M.; Yasukawa, K. J. Pharm. Nutr. Sci. 2012, 2, 127.

15. Park, Y. K.; Paredez-Guzman, J. F.; Aguiar, C. L.; Alencar, S. M.; Fujiwara, F. Y.; J. Agr. Food Chem. 2004, 52, 1100; Midorikawa, K.; Banskota, A. H.; Tezuka, Y.; Nagaoka, T.; Matsushige, K.; Message, D.; Huertas, A. G.; Kadota, S.; Phytochem. Anal. 2001, 12, 366.

16. Kasote, D.; Suleman, T.; Chen, W.; Sandasi, M.; Viljoen, A.; Van Vuuren, S.; Biochem. Syst. Ecol. 2014, 55, 156; Zhang, J.; Cao, X.; Ping, S.; Wang, K.; Shi, J.; Zhang, C.; Zheng, H.; Hu, F.; Evidence-Based Complementary Altern. Med. 2015, ID 307594

17. Szliszka, E.; Mertas, A.; Czuba, Z. P.; Wojciech, K.; Evidence-Based Complementary Altern. Med. 2013, ID 735176.

18. Moura, M. C. S.; Lopes, A. N. C.; Moita, G. C.; Moita Neto, J. M.; Quim. Nova 2006, 29, 429. 